\title{
Intercomparison Study on Accurate Mass Measurement of Small Molecules in Mass Spectrometry
}

\author{
Anthony W. T. Bristow and Kenneth S. Webb \\ LGC Limited, Middlesex, United Kingdom
}

The results from an intercomparison of accurate mass measurement of a small molecule (molecular weight $475 \mathrm{Da}$ ) across a broad range of mass spectrometers are reported. The intercomparison was designed to evaluate the relative capabilities and the optimum methodology of the diverse range of mass spectrometers currently used to record accurate mass measurements. The data will be used as a basis for developing guidance on accurate mass measurement. The need for guidance has resulted from the continued growth in the use of accurate mass measurements for assignment of elemental formula in the chemical and biochemical industries. This has been fuelled by a number of factors and includes the rapid pace of instrument development, which has enabled accurate mass measurements to be made in a less costly, yet robust fashion. The data from the intercomparison will allow us to compare those protocols that produced excellent accuracy and precision with those that produced poorer accuracy and/or precision for each type of mass spectrometer. The key points for best practice will then be established from this comparison for each type of mass spectrometer and accurate mass measurement technique. A compound was sent to the participating laboratories (in the UK, Europe, and USA), the identity of which was not revealed. Each laboratory was asked to record a minimum of five repeat mass measurements of the molecular species using their local protocols and their preferred ionization technique or techniques. To the best of our knowledge there were no interfering (unresolved) ions that originated from the sample. A questionnaire was also completed with the experimental work. The information from the questionnaires was used to evaluate the protocols used to record the measurements. Forty-five laboratories have reported their results. To summarize the performance of mass spectrometers in the intercomparison, magnetic sector field mass spectrometers used in peak matching mode and FTMS reported the highest mean mass measurement accuracy (88 and 83\%, respectively, achieved $\leq 1 \mathrm{ppm}$ ). Magnetic sector field mass spectrometers used in voltage scanning produced $60 \%$ of the mean mass measurements with accuracy $\leq 1 \mathrm{ppm}$. Magnetic sector field mass spectrometers used in magnet scanning modes, quadrupole-TOF and TOF instruments generally achieved mean mass measurement accuracy between 5 and $10 \mathrm{ppm}$. The two low resolution triple quadrupoles used in the inter-comparison produced mean mass measurement accuracy of $<2 \mathrm{ppm}$. The precision of the data from each instrument and experiment type is an important consideration when evaluating their relative capabilities. Using both the precision and accuracy, it will be possible to define the uncertainty associated with the elemental formulae derived from accurate mass measurements. Therefore, a thorough statistical evaluation of the data is underway and will be presented in a subsequent publication. (J Am Soc Mass Spectrom 2003, 14, 1086-1098) (c) 2003 American Society for Mass Spectrometry

A ccurate mass measurement of small molecules is used to determine elemental formulae. The better the accuracy the less the ambiguity [1]. In general, accurate mass measurement is non-ambiguous up to 300 daltons $(\mathrm{Da})$ due to the small number of potential molecular formulae. With increasing mass to

Published online August 28, 2003

Address reprint requests to Dr. A. W. T. Bristow, LGC Limited, Queens Road, Teddington, Middlesex TW110LY, UK. E-mail: tony.bristow@ lgc.co.uk charge ratio $(\mathrm{m} / \mathrm{z})$ the number of possible formulae dramatically increases making identification more and more difficult [2]. There are many applications for which accurate mass measurement is required. These include identification of the components of mixtures, biologically important molecules, metabolites, environmental pollutants, patent protection, synthetic chemistry and polymer characterisation. In the light of this broad application range and the continued commercialisation of new instruments capable of accurate mass measurements, the American Society of Mass Spectro- 
metry (ASMS) have began to develop guidance on the use of high resolution mass spectral data for confirmation of elemental formulae $[1,2]$.

As early as 1927, Aston made measurements to an accuracy of 1 part in 10,000 (100 ppm) using a magnetic sector field mass spectrometer with a resolving power of 600 [3]. From the late 1950s, high resolution experiments and accurate mass measurement for the determination of elemental formulae were developed and demonstrated using double focusing magnetic sector field mass spectrometers. In 1959 John Beynon published a report demonstrating that the $\mathrm{m} / \mathrm{z}$ value of an ion can be measured with sufficient accuracy so as to determine its elemental composition [4]. A variety of techniques were established to achieve high accuracy using double focusing magnetic sector field mass spectrometers. These were peak matching, pioneered by Nier [5-7], dynamic voltage (or acceleration voltage) scanning and dynamic magnet scanning [8].

The technology of accurate mass measurement using magnetic sector field mass spectrometers continued to evolve. Klaus Bieman described the use of photographic plates and a Mattauch-Herzog geometry sector field instrument for precise measurement of line position [9]. Two important developments were presented at the 13thAnnual Conference on Mass Spectrometry and Allied Topics in St. Louis in 1965. Campbell and Halliday provided a theoretical evaluation by relating statistical variation in peak position to ion abundance [10]. Green et al. demonstrated the digitization of spectra recorded electronically on magnetic tape for accurate mass measurement, using an MS9 mass spectrometer [11].

Accurate mass measurements are no longer restricted to double focusing magnetic sector field instruments and are now carried out using a variety of mass spectrometers. Fourier transform mass spectrometry (FTMS) offers the highest mass resolution and mass accuracy of any mass spectrometer [12]. FTMS is routinely used for accurate mass measurement with mass resolving power in excess of $1 \times 10^{6}$ and mass accuracy of $1 \mathrm{ppm}$ [13]. An excellent example of the capability of FTMS is the resolution of 500 peaks in the spectrum of a raw diesel feedstock using a 5.6 tesla instrument. All of the components were identified with a mass accuracy of $<0.3 \mathrm{ppm}$ [13]. In his FTMS tutorial, Jon Amster has highlighted the key paramaters to consider for accurate mass measurement on the FTMS [14].

Eckers et al. [15] demonstrated mass measurement accuracy of $\leq 4 \mathrm{ppm}$ using an orthagonal acceleration time of flight (TOF) mass spectrometer and a lock (reference) mass ion. A number of reports have demonstrated that high accuarcy mass measurements can be recorded using quadrupole-TOF mass spectrometers. Wolff et al. achieved mass measurement accuracy to within $5 \mathrm{mDa}$ for ions in the $\mathrm{m} / \mathrm{z}$ range 74 to 423 [16]. Blom has also demonstrated mass measurement accuracy of $<3 \mathrm{ppm}$ when using a quadrupole-TOF in conjunction with a Z-Spray source [17]. Mass accuracy of better than 2 ppm has been demonstrated by Rich- ards when using a quadrupole-TOF mass spectrometer. Two lock mass ions were employed and a lock mass/ analyte ion abundance ratio of 1 was recommended. The data was used to identify the oxidative metabolites of the antihypertensive Bufuralol [18].

High accuracy mass measurement has not been restricted to instruments that are capable of operating at high resolution. As early as 1965 it was understood the position of peaks on the mass scale could be measured at low resolution with ppm accuracy provided they are present at adequate abundance and no interferences are present. An internal calibrant was necessary and this was demonstrated by Haddon and Lukes in 1974 [19].

Though not usually considered as tools for accurate mass measurement, low resolution quadrupole, triple quadrupole, and quadrupole ion trap mass spectrometers have been applied to such measurements. Haddon et al. demonstrated the use of a single quadrupole instrument for accurate mass measurement and described a novel peak-finding algorithm that produced improved mass accuracy [20]. Green and coworkers have also reported mass measurement accuracy to within $5 \mathrm{ppm}$ of the correct mass value using a single quadrupole [21]. Mass measurement accuracy of 10 ppm using a quadrupole ion trap has also been reported [22].

In $2001 \mathrm{O}^{\prime}$ Connor and Sargent at LGC carried out a study to evaluate the measurement issues arising from the wide range of accurate mass measurement applications, in order to obtain independent views on issues such as the scope of the topic, problem areas, and ways in which they can be addressed [23]. The study (carried out using literature review, interviews, and a forum meeting) highlighted the continued growth in the use of accurate mass measurements in the chemical and biochemical industries. This has been fuelled by a number of factors and includes the rapid pace of instrument development, which has enabled accurate mass measurements to be made in a less costly, yet robust fashion. It was noted that the pace at which this new technology is being introduced might potentially outpace the development of expertise for adequate validation.

The main conclusions arising from the 2001 study were:

- A lack of understanding, particularly amongst newer users, of basic terminology and definitions.

- The limitations of specific equipment for particular purposes.

- The requirement for appropriate selection of both external and internal calibrants.

- The distinction between measurements for the confirmation of a known species and the identification of an unknown. 
- The need to have an overall uncertainty estimate for the data; the reproducibility of repetitive accurate mass determinations and the accuracy achievable when measuring a truly unknown species, is often confused.

- The errors which can arise from species such as adducts formed in the mass spectrometer itself and interfering ions from other sources.

- The rapidly increasing requirement to make measurements on mixtures and the issues arising from the use of separation techniques.

In the light of the conclusions from the study, it was clear that there is a need to investigate the various capabilities of the different instruments, together with their optimum methodology and to provide guidance on accurate mass measurement. Therefore, an intercomparison study to evaluate the variation in accurate mass measurement and to understand the relative capabilities of each type of mass spectrometer was coordinated by LGC in 2002. The data and conclusions from the study are presented in this paper. As a result of the identification of the key measurement issues associated with accurate mass measurement, an accurate mass measurement guide is being developed which will tackle these key measurement issues and use the conclusions from the intercomparison to establish the best practice for each type of mass spectrometer.

\section{Experimental}

A single unknown compound (nominal molecular weight $475 \mathrm{Da}$ ) was circulated to the participating laboratories, together with a questionnaire to be completed with the experimental work. The questionnaire was designed to evaluate the protocols used to make the measurements. The compound provided was a research material that had not previously been available to the participants, with the exception of the sample provider. The participants were asked to make five repeat mass measurements (on separate days where possible) of the molecular ion using their local protocols and their preferred ionization technique or techniques. A typical $70 \mathrm{eV}$ electron ionization spectrum of the intercomparison compound, supplied by one of the participants, is shown in Figure 1.

\section{Results and Discussion}

The data is discussed in terms of the ion measured, the type of mass spectrometer used, the local protocols employed and the time period in which the measurements were made. The study instructions requested that the mass measurements should be recorded on separate days, but this was not possible for all the laboratories that contributed data to the study. The five

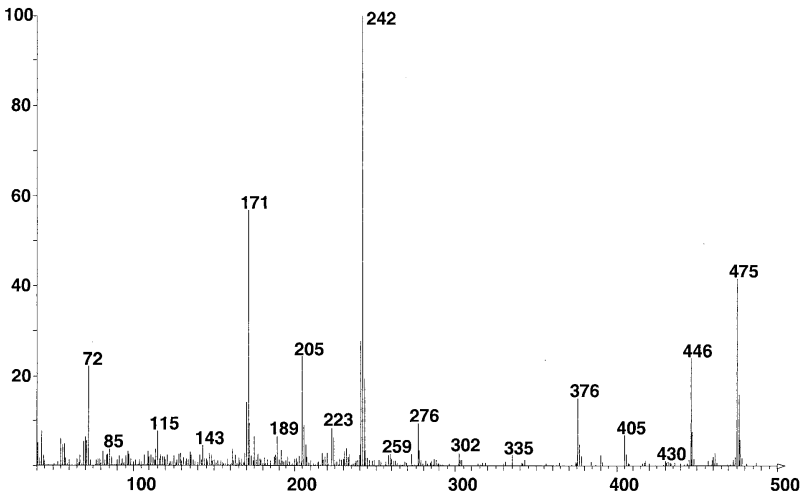

Figure 1. A typical $70 \mathrm{eV}$ electron ionization spectrum of the intercomparison compound supplied by a participant.

measurements were recorded on a single day or in a time period varying between two days and eight weeks.

Each laboratory was provided with a unique identification number. For the purpose of reporting the data, a number of laboratories have been given a letter extension to their unique number (e.g.; $2 a$ and $2 b$ ). This was required, as many laboratories have provided multiple sets of measurements. These include the use of more than one type of mass spectrometer, recording more than one set of data on a single mass spectrometer, using multiple ionization techniques, or data recording by multiple users. All instruments are referred to in generic terms with no reference to any specific mass spectrometer manufacturer.

Forty-five laboratories have reported data for the intercomparison. This translates to well over 100 individual data sets as many laboratories have provided multiple measurements. The results are shown in Figures $2,3,4$, and 5 . The figures show the mean of the five mass measurements, with the error bars representing the range (highest value and the lowest value). For comparison with the reported data, the expected $\mathrm{m} / \mathrm{z}$ of the measured ions, the $\pm 1 \mathrm{ppm}$ range, the $\pm 5 \mathrm{ppm}$ range, and $\pm 10 \mathrm{ppm}$ range are also plotted. These have been calculated to take into account the mass of an electron (i.e., the mass of an electron, $0.00055 \mathrm{Da}$, has been removed from the calculation of expected $m / z$ of the ions measured and from the various accuracy ranges). The data supplied by the contributors is plotted as reported, with no correction for the mass of an electron.

\section{The Measurement of the $\mathrm{m} / \mathrm{z} 475$ Ion}

With only two exceptions all the data reported for the mass measurement of the molecular $\left(\mathrm{M}^{+} \bullet\right)$ ion, $m / z 475$, was recorded using double focusing magnetic sector field instruments, utilizing either electron ionization (EI) or fast atom bombardment (FAB) ionization. The exceptions to this were was laboratory 44 and laboratory 53. Laboratory 44 employed a TOF combined with secondary ion mass spectrometry (SIMS) and Labora- 


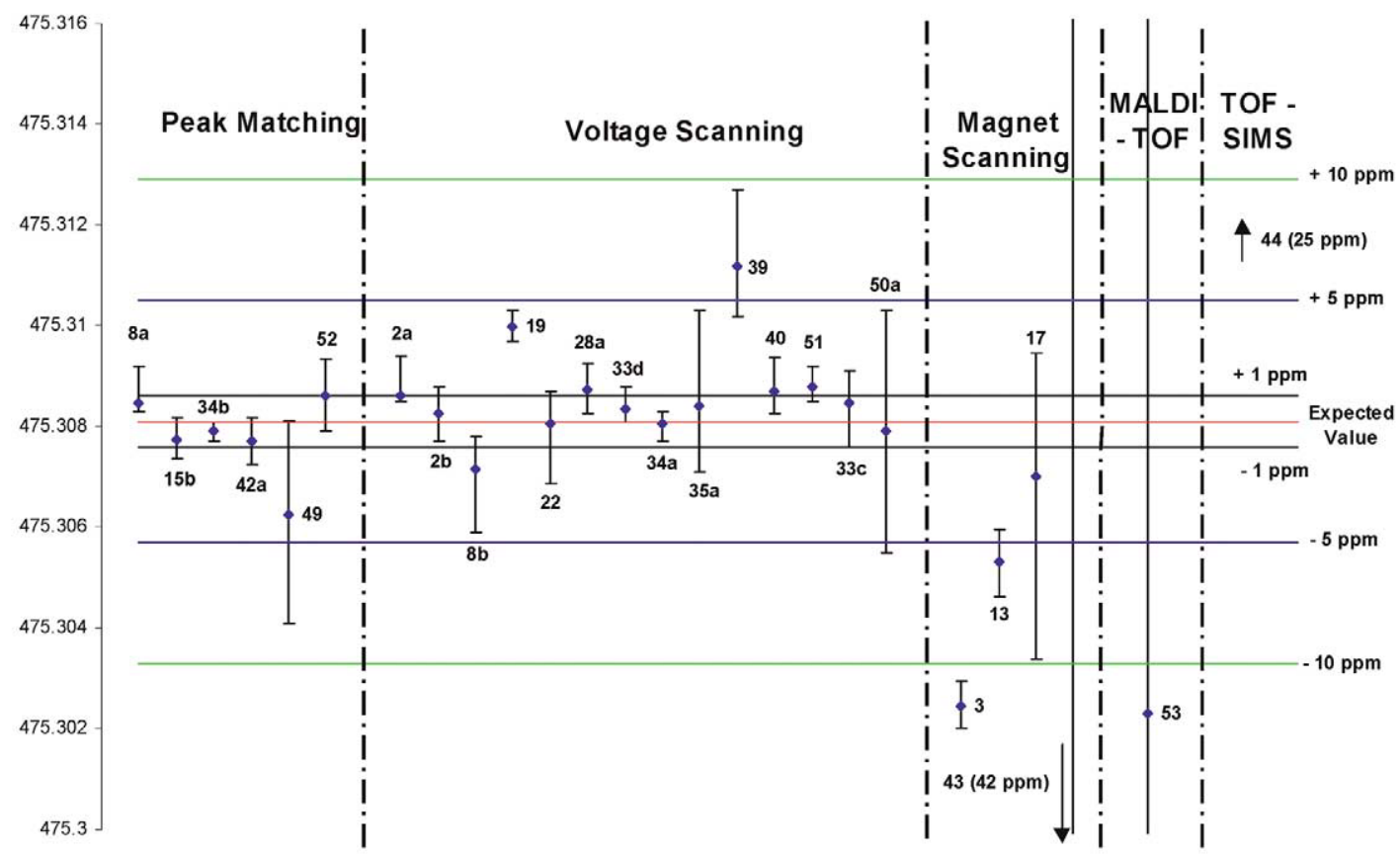

Figure 2. Results from the mass measurement of the $\mathrm{M}^{+}{ }^{\bullet}$ ion at $m / z 475( \pm 10 \mathrm{ppm}$ view).

tory 53 reported data recorded using MALDI-TOF. The data is summarized in Figure 2.

\section{Double Focusing Magnetic Sector Field Instruments and Peak Matching}

With the exception of laboratory 49 (4 ppm), all of the laboratories that employed the peak matching technique $(8 \mathrm{a}, 15 \mathrm{~b}, 34 \mathrm{~b}, 42 \mathrm{a}$, and 52$)$ reported data with a mean mass measurement of $\leq 1 \mathrm{ppm}( \pm \mathrm{m} / \mathrm{z} 0.00048)$. Instrument resolving power between 3000 and 12,000 (10\% valley definition-two peaks of equal abundance are considered to be resolved when they are separated by a valley which is $10 \%$ of the height of either peak and which is made up from 5\% contribution from each component) was reported. Laboratories $15 \mathrm{~b}$ and 49 recorded all measurements on a single day. The remainder of the laboratories reported measurements recorded over time periods ranging from 2 to 28 days. All of the laboratories used a lock (reference) mass ion that was no greater than $44 \mathrm{~m} / \mathrm{z}$ away from the analyte ion (lowest $m / z$ 431, highest $m / z$ 481). The lock (reference) mass ions were generated by EI of either perfluorotributylamine (PFTBA) or perfluorokerosene (PFK) intro-

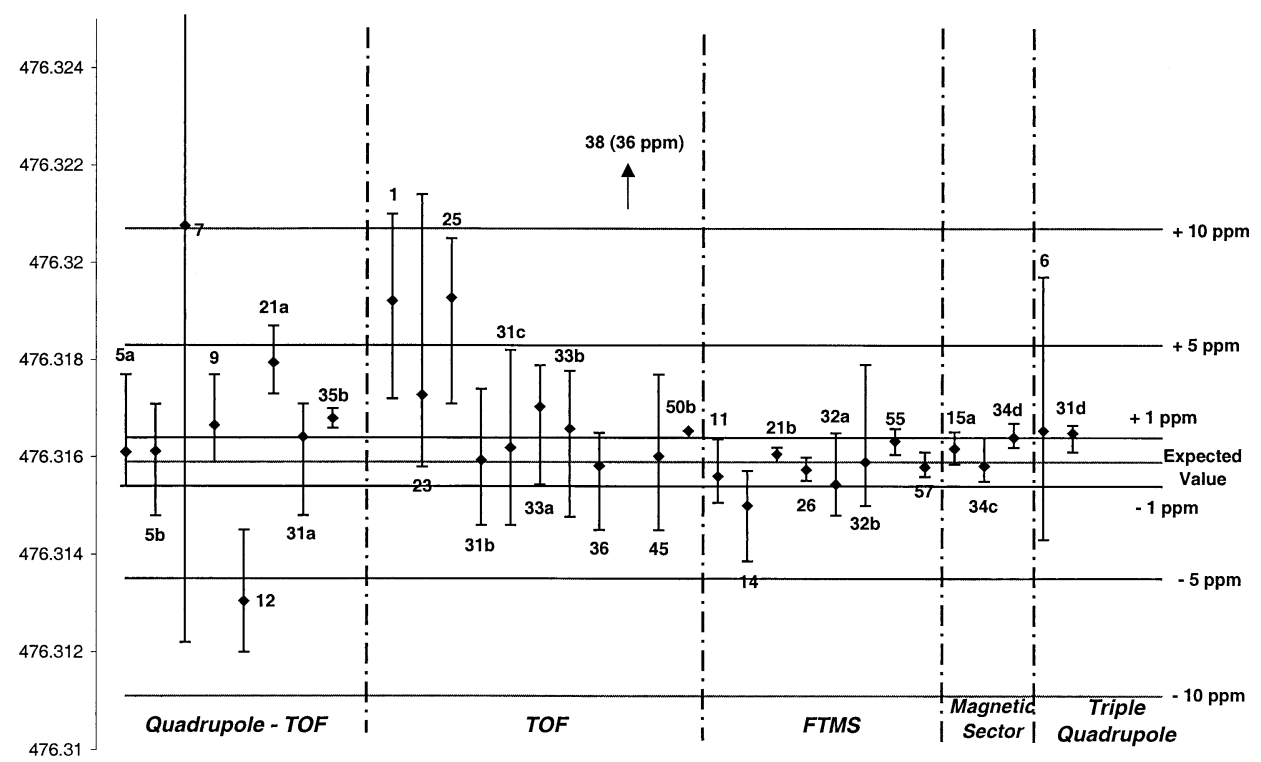

Figure 3. Results from the mass measurement of the $[\mathrm{M}+\mathrm{H}]^{+}$ion at $m / z 476( \pm 10 \mathrm{ppm}$ view). 


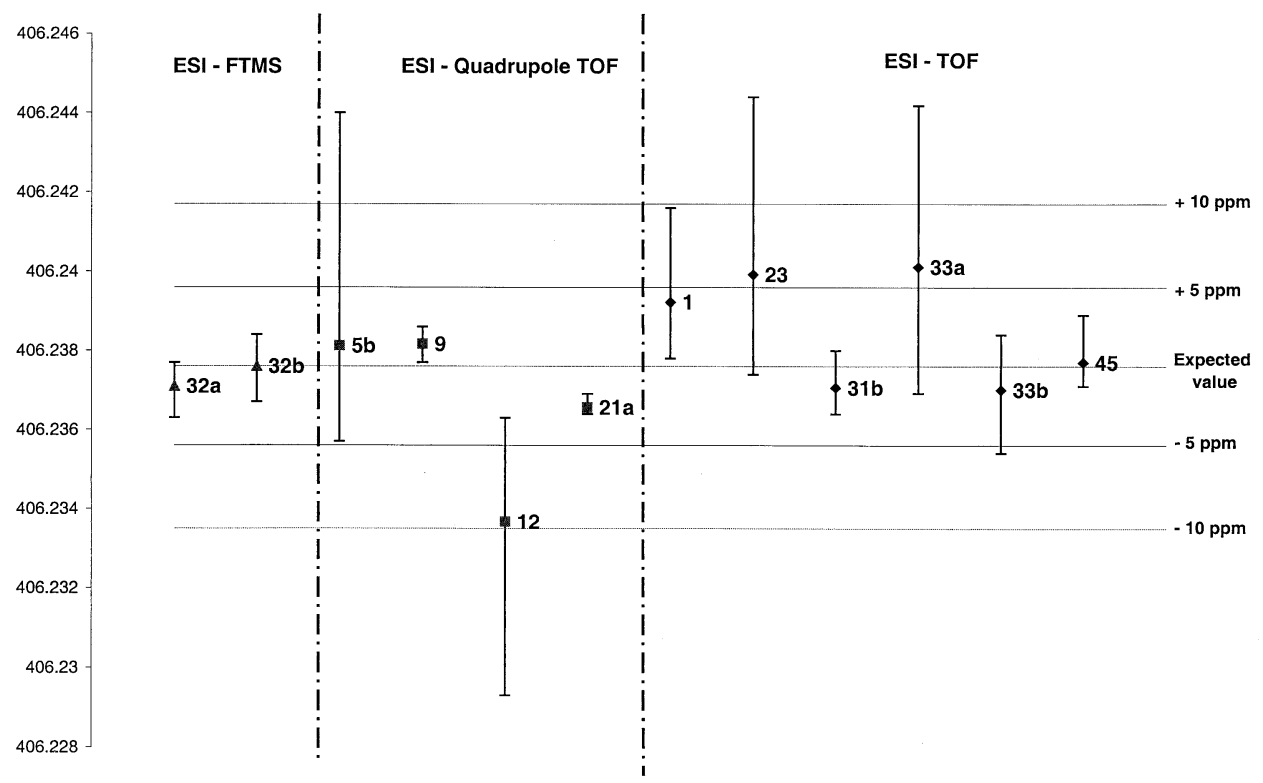

Figure 4. Results from the mass measurement of the $\left[\mathrm{M}+\mathrm{H}-\mathrm{C}_{5} \mathrm{H}_{10}\right]^{+}$ion at $m / z 406$.

duced via a leak valve and present throughout the measurements. Laboratory 42a used either PFK ions or $\mathrm{m} / \mathrm{z} 472$ generated from vitamin $\mathrm{E}$ acetate which was premixed with the analyte on the solids probe. The abundance ratio of $\mathrm{m} / \mathrm{z} 475$ to the lock (reference) mass ion ranged from 5:1 through to 1:2. From the limited data, there is no evidence to suggest improved mass accuracy in the measurements made on a single day compared to those recorded over a longer time period. For example, if we compare $15 \mathrm{~b}$ and $34 \mathrm{~b}$. This provides confidence that the protocols employed are robust over time and were consistently applied.

A number of subtle variations on the peak matching technique were employed. Laboratory $15 \mathrm{~b}$ initially car- ried out a two point accelerating voltage $(\mathrm{V})$ calibration using $\mathrm{m} / \mathrm{z} 218$ and 263. The sample and lock (reference) mass $(\mathrm{m} / \mathrm{z} 414)$ were then introduced. The sample $\mathrm{m} / \mathrm{z}$ is then noted into the data system and then $\mathrm{V}$ is automatically adjusted to overlap the sample and lock mass ion and the calculation and measurement are reported by the data system. Laboratory 52 also used an automated procedure, which uses two lock (reference) masses. The data system selects the first lock (reference) mass (A), carries out an electric calibration between it and the second lock (reference) mass (B). It then measures from (A) to the sample ion and from the sample ion to (B). An average is taken of the two measurements and this result is placed in a table $\mathrm{n}$ times ( $n$ is typically set to

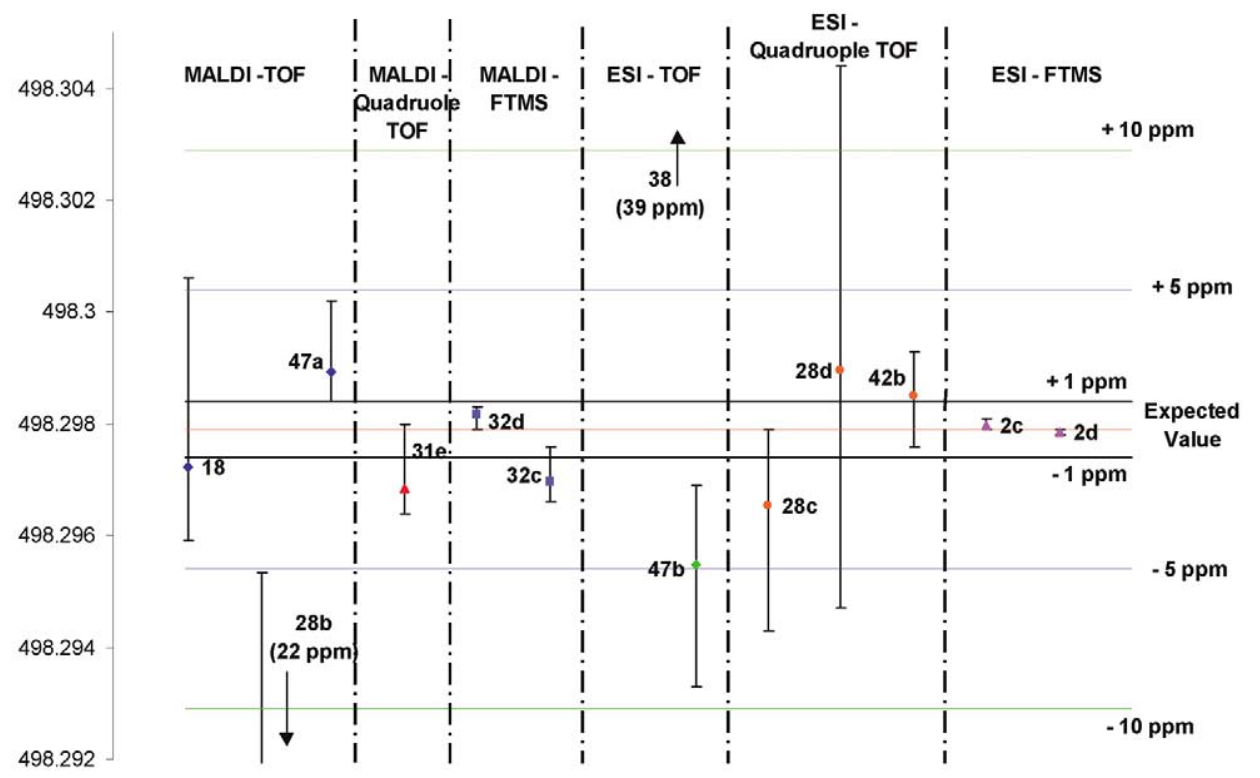

Figure 5. Results from the mass measurement of the $[\mathrm{M}+\mathrm{Na}]^{+}$ion at $m / z 498( \pm 10 \mathrm{ppm}$ view). 
Table 1. The percentage of mean mass measurements at $\leq 10 \mathrm{ppm}, \leq 5 \mathrm{ppm}$, and $\leq 1$ ppm for each type of mass spectrometer

\begin{tabular}{|c|c|c|c|}
\hline \multirow[b]{2}{*}{ Instrument type } & \multicolumn{3}{|c|}{$\%$ of mean mass measurements } \\
\hline & $\leq 10 \mathrm{ppm}$ & $\leq 5 \mathrm{ppm}$ & $\leq 1 \mathrm{ppm}$ \\
\hline \multicolumn{4}{|c|}{ Double focusing magnetic sector field (measuring $\mathrm{m} / \mathrm{z} 475$ and 476) } \\
\hline Peak matching $n=8$ & 100 & 100 & 88 \\
\hline Dynamic voltage scanning $n=15$ & 100 & 93 & 60 \\
\hline Dynamic magnet scanning $n=4$ & 50 & 25 & 0 \\
\hline FTMS (measuring $m / z 476$ and 498) $n=12$ & 100 & 100 & 83 \\
\hline Quadrupole-TOF (measuring $m / z 476$ and 498) $n=12$ & 100 & 83 & 25 \\
\hline TOF (measuring $m / z 475,476$, and 498 ) $n=17$ & 76 & 65 & 24 \\
\hline Triple-Quadrupole (measuring $\mathrm{m} / z$ 476) $n=2$ & 100 & 100 & 0 \\
\hline
\end{tabular}

10). Therefore each of the five results reported by laboratory 52 are a mean of the 10 individual measurements. Laboratory $42 \mathrm{a}$ initially calibrated the peak matching unit using $\mathrm{m} / \mathrm{z} 181$ and 193 prior to the mass measurement of $\mathrm{m} / \mathrm{z} 475$.

\section{Double Focusing Magnetic Sector Field Instruments and the Dynamic Scanning Techniques}

Eighteen laboratories employed a dynamic scanning method with EI or FAB. The data from these laboratories was acquired using two different techniques, the magnet scan and the dynamic voltage scan.

Dynamic voltage scan. Laboratories $2(\mathrm{a}+\mathrm{b}) 8 \mathrm{~b}, 19,22$, 28a, 33(c + d), 34a, 35a, 39, 40, 50a, and 51 used this type of experiment. Instrument resolving power between 3000 and 10,000 (10\% valley definition) was reported. With the exception of laboratory 39 (mean $=6.5 \mathrm{ppm})$, all laboratories reported data with a mean mass measurement accuracy of less than 5 ppm $( \pm 0.00238 \mathrm{Da})$. Laboratories $2(\mathrm{a}+\mathrm{b}), 22,33(\mathrm{c}+\mathrm{d}), 34 \mathrm{a}, 35 \mathrm{a}$, and 50a reported data with a mean mass measurement accuracy of $\leq 1 \mathrm{ppm}( \pm \mathrm{m} / \mathrm{z} 0.00048 \mathrm{Da})$. Laboratories $2(\mathrm{a}+\mathrm{b}), 33$ $(c+d)$, and 50a recorded all measurements in a single day (two sets of five, on two different days for laboratory 2). All other laboratories recorded their data over a time period varying from 2 to 28 days. Once again, the data suggests that the protocols employed were not affected by the time period over which the measurements were recorded (compare 28a and 2a).

All the laboratories employed very similar experimental protocols. In nearly all cases a narrow range internal calibration was used to make the measurements. For the majority of the laboratories using EI, between 1 and 3 ions from PFK were used for the internal calibration with the PFK introduced at the same time as the sample. The $m / z$ range for internal calibration ion was no greater than $\pm 56 \mathrm{~m} / z$ units from the $\mathrm{m} / \mathrm{z}$ 475 ion $(m / z 431-m / z 531)$ for all laboratories. In the experiment carried out by laboratory $35 \mathrm{a}, \mathrm{PFK}$ is present throughout the experiment and the sample was introduced using the solids probe. After the sample had evaporated from the probe and the experiment was complete, a single scan from PFK (recorded before the sample is observed) is used to create an accelerating voltage external calibration. The $m / z$ of the sample molecular ion and its fragments are measured using this calibration.

For the two laboratories employing FAB (33c and 50a), two ions from poly(ethyleneglycol) 600 that bracketed $m / z 475(m / z 459$ and $m / z 503)$ were used for internal calibration. Laboratory $33 \mathrm{c}$ used a subtly different protocol, where the internal calibrant is applied to one target and the analyte is applied to the second target of a dual target probe. During acquisition the shaft of the probe is rotated between the two targets providing a series of scans with interleaved reference and analyte spectra. When processing, all scans are averaged to create a single spectrum of internal calibrant and analyte ions. Laboratories $8 b, 22,28 a, 33 c$, $33 \mathrm{~d}, 34 \mathrm{a}, 40$, and 51 maintained the analyte to calibrant ion abundance ratio between 5:1 and 1:2.

Magnet scan. Laboratories 3, 13, 17, and 43 used a magnet scan to record their accurate mass measurements. Resolving power between 5000 and 12,000 (10\% valley definition) was reported. The results from this approach produced much greater variation in mean mass measurement accuracy, as low as 3 ppm (laboratory 17) to a high of $42 \mathrm{ppm}$ (laboratory 43). An explanation for this variability is not readily apparent from the questionnaires.

Laboratory 43 used a broad range internal calibration $(\mathrm{m} / \mathrm{z}$ 32-500) to make the measurements, which allowed the measurement of both the $m / z 475$ ion and a number of the fragment ions. PFK was used for internal calibration and this was introduced at the same time as the sample. Laboratory 13 also used PFK for internal calibration and for these experiments appropriate pairs of ions that bracketed each analyte and fragment ion to be measured (e.g., $m / z 169$ and $m / z 181$ for the fragment at $m / z$ 171) were employed to create the narrow range internal calibrations. Laboratory 3 carried out a narrow range external calibration $(m / z 430-500)$ followed by a lock (reference) mass correction $(\mathrm{m} / \mathrm{z} 481$ from PFK) to mass measure the $m / z 475$ ion. For Laboratory 17 
analysis commences with only PFK present in the mass spectrometer. About 30 scans were recorded and these are used to create an external calibration. Without stopping the acquisition, the sample was introduced and PFK is present to provide lock (reference) masses over the full scan range. For all four laboratories the measurements were recorded over a long time period (17 days to 8 weeks).

\section{TOF-SIMS}

Laboratory 44 reported the mass measurement of the $\mathrm{m} / \mathrm{z} 475$ ion using TOF-SIMS and the mean mass measurement accuracy was $25 \mathrm{ppm}( \pm \mathrm{m} / \mathrm{z}$ 0.01188). Calibration was carried out prior to each measurement using known ions produced during the SIMS process, but only over the range $m / z 15$ to 203. A lock (reference) mass correction was not employed. The mass accuracy achieved met the expectation of the data provider, for their routine applications.

\section{MALDI-TOF}

Unusually, laboratory 53 reported the mass measurement of the $m / z 475$ ion using MALDI-TOF. The mean mass measurement accuracy was $12 \mathrm{ppm}( \pm \mathrm{m} / \mathrm{z}$ $0.0057)$. Prior to the measurements an aliquot $(1.0 \mu \mathrm{L})$ of a $10 \mathrm{mg} / \mathrm{mL}$ solution of the sample was mixed with $1 \mu \mathrm{L}$ of PEG1000 $(10 \mathrm{mg} / \mu \mathrm{L})$ and $1 \mu \mathrm{L}$ dithranol $(10$ $\mathrm{mg} / \mu \mathrm{L})$ in a tube. One $\mu \mathrm{L}$ of the mixture was placed onto the MALDI target and then allowed to dry prior to analysis. Internal calibration, using the PEG 9-mer $\left([\mathrm{M}+\mathrm{Na}]^{+}=437.2360\right)$ and $10-$ mer $\left([\mathrm{M}+\mathrm{Na}]^{+}=\right.$ 481.2622) signals, was used to record the mass measurements.

\section{The Measurement of the $m / z 476$ Ion, the $m / z 498$ Ion, and the $m / z 406$ ion}

The accurate mass measurement of the $[\mathrm{M}+\mathrm{H}]^{+}$ion $\left(m / z\right.$ 476), the $[\mathrm{M}+\mathrm{Na}]^{+}$ion $(m / z 498)$, and the $[\mathrm{M}+\mathrm{H}$ $\left.-\mathrm{C}_{5} \mathrm{H}_{10}\right]^{+}$ion $(m / z 406)$, was carried out using double focusing magnetic sector field, triple quadrupole, TOF, FTMS and quadrupole-TOF mass spectrometers combined with electrospray ionization (ESI). Laboratories 7, $32(\mathrm{a}+\mathrm{b}), 34 \mathrm{~d}$ and $50 \mathrm{~b}$ recorded their measurements in a single day. All other laboratories made measurements over a period of three days to eight weeks. The results will now be discussed in terms of the mass spectrometer used to make the measurement.

\section{Quadrupole-TOF}

Eight laboratories reported the mass measurement of the $\mathrm{m} / \mathrm{z} 476$ ion with quadrupole-TOF and ESI, with instrument resolving power ranging from 9000 to 16,500 reported (full width at half maximum [FWHM] definition-where the width of the peak at half its height is used to calculate resolution). Three laboratories $5(\mathrm{a}+\mathrm{b})$ and 31a reported a mean mass measurement accuracy of $\leq 1 \mathrm{ppm}$ and laboratories 9, 21a, and $35 \mathrm{~b}$ reported a mean mass measurement accuracy of $<5$ ppm (Figure 3). Where a mass accuracy of $<5$ ppm was achieved, all contributors employed 1 or 2 lock (reference) mass ions and had carried out an external calibration on the day of the measurement or no longer than two days prior to each measurement. One exception was laboratory 5a, which employed an external calibration of the TOF that was between 16 and 17 weeks old depending on the date of the measurement.

The lock (reference) mass ions employed for the measurements were in the range $m / z 307$ to 734 . At this stage of the data evaluation, no attempt has been made to correlate mass accuracy with the $\mathrm{m} / \mathrm{z}$ difference between the analyte and lock (reference) ions. The highest analyte to lock (reference) mass ion abundance ratio was 20:1, but the majority lay between 3:1 and 1:3. Three laboratories also reported the simultaneous measurement of the $m / z 406$ ion (5b, 9, and 21a) and mean mass measurement accuracy of $<5$ ppm (Figure 4 ).

Laboratories 7 and 12 reported mean mass measurement accuracy between 5 and 10 ppm. Laboratory 7 achieved this using a lock (reference) mass $(\mathrm{m} / \mathrm{z} 556)$, with an analyte to lock (reference) mass ion abundance ratio between 0.4:1 and 3:1 (Figure 3). Laboratory 12 achieved their accuracy without the use of a lock mass (reference) ion. Laboratory 12 also reported mean mass measurement accuracy of $9 \mathrm{ppm}$ for the $m / z 406$ ion (Figure 4), the mass measurements were made simultaneously with the $\mathrm{m} / \mathrm{z} 476$ ion. The greater variability in the data from laboratory 7 , cannot be explained by reference to the information in the questionnaire, as the protocols used matched those employed by laboratories that reported both greater accuracy and precision (compare laboratory 7 and laboratory 31a).

Laboratories $28(c+d)$ and $42 b$ reported mean mass measurement accuracy of $<5$ ppm for the $[\mathrm{M}+\mathrm{Na}]^{+}$ ion at $\mathrm{m} / \mathrm{z} 498$ (Figure 5). Of those laboratories, 28c used an internal calibration procedure to make the measurements and the internal calibrant was introduced by mixing with the sample. The internal calibrant ions employed for the measurement were $\mathrm{m} / \mathrm{z} 437$ and 525 and the analyte to internal calibrant ion abundance ratio lay between 5:1 and 1:3. For the measurements reported by laboratory $28 \mathrm{~d}$, only an external instrument calibration (m/z 100-2000) was employed, which was carried out on the same day of each measurement reported. The data for $28(\mathrm{c}+\mathrm{d})$ was recorded in a single day. Laboratory $42 \mathrm{~b}$ reported mean mass measurement accuracy of $1 \mathrm{ppm}$ for the $\mathrm{m} / \mathrm{z} 498$ ion, using a lock (reference) mass $(\mathrm{m} / \mathrm{z}$ 662) and an analyte to lock (reference) mass ion abundance ratio between 20:1 and 1:2. The mass measurements were recorded on a single day, with an external calibration recorded on the same day. 
Table 2. The participants in the accurate mass measurement intercomparison

\begin{tabular}{|c|c|}
\hline Name & Organization \\
\hline Alison Ashcroft & School of Biochemistry \& Molecular Biology, University of Leeds \\
\hline Stephen Baldwin & AstraZeneca \\
\hline Perdita Barran & SIRCAMS, University Of Edinburgh \\
\hline Claire Beaumont & GlaxoSmithkline Pharmaceuticals \\
\hline David Bendell & CHEMISPEC, University of Sunderland \\
\hline John Bickerton & The University Of Warwick \\
\hline Malcolm Clench & School of Science and Mathematics, Sheffield Hallam University \\
\hline Alex Colburn & Institute of Mass Spectrometry and Department of Chemistry, The University Of Warwick \\
\hline Sarah Dykes & Pfizer Global R\&D \\
\hline Laurent Fay & Nestlé Research Centre, Nestec Limited \\
\hline Roderick Ferguson & School of Engineering and Physical Sciences Chemistry, Heriot-Watt University \\
\hline Betty Flitcroft & Avecia \\
\hline Trevor Gibson & Cubist Pharmaceuticals (UK) Limited \\
\hline John Gilbert & Jeol UK Limited \\
\hline Ian Gilmore & National Physical Laboratory \\
\hline Padraig Glynn & Elan Corporation \\
\hline Mark Gosling & AstraZeneca \\
\hline Ian Gould & Kodak Limited \\
\hline Brian Green & Waters Corporation MS Technologies Centre, Micomass UK Limited \\
\hline Chris Harbach & M-Scan Limited \\
\hline Joan Hague & School of Science and Mathematics, Sheffield Hallam University \\
\hline Neville Haskins & Consultant \\
\hline Julie Herniman & University Of Southampton \\
\hline Andrew Hoteling & Eastman Kodak Company \\
\hline Ann Hunter & EPSRC National Mass Spectrometry Service Centre, Swansea \\
\hline Andrew Illsley & Shimadzu Biotech \\
\hline Tom Jackson & Eastman Kodak Company \\
\hline Jackie Jarvis & Bruker Daltonics \\
\hline Keith Jennings & Consultant \\
\hline Mike Jones & Department of Chemistry,University of Durham \\
\hline George Keenan & Scottish Water \\
\hline Steve Lane & CASS, GlaxoSmithKline, UK \\
\hline John Langley & University Of Southampton \\
\hline Pat Langridge-Smith & SIRCAMS, University Of Edinburgh \\
\hline Bill Leavens & CASS, GlaxoSmithKline, UK \\
\hline Mervyn Lewis & IACR \\
\hline Steve Lock & Applied Biosystems/MDS SCIEX \\
\hline Tony Mallet & School of Chemical and Life Sciences, University Of Greenwich \\
\hline Peter Marshall & CASS, GlaxoSmithKline, UK \\
\hline Rachel Martin & Shimadzu Biotech \\
\hline Peter Maziarz & Bausch and Lomb \\
\hline Mike McCullagh & Waters Corporation MS Technologies Centre, Micromass UK Limited \\
\hline Bryan McCullough & SIRCAMS, University Of Edinburgh \\
\hline George McLeod & Syngenta \\
\hline John Monaghan & School of Chemistry, University Of Edinburgh \\
\hline Bill Morden & LGC Limited \\
\hline Mike Morris & Waters Corporation MS Technologies Centre, Micromass UK Limited \\
\hline Bill Nichols & Eastman Kodak Company \\
\hline Carla Owen & Applied Biosystems/MDS SCIEX \\
\hline Hema Pancholi & AstraZeneca \\
\hline George Perkins & Pfizer Global R\&D \\
\hline Russel Pickford & UMIST \\
\hline Nick Polfer & SIRCAMS, University Of Edinburgh \\
\hline Jens Griep-Ramming & ThermoFinnigan MAT GmbH \\
\hline Don Richards & Pfizer Global R\&D \\
\hline Stone. D. H. Shi & CASS UM, GlaxoSmithKline (USA) \\
\hline Bridget Stein & EPSRC National Mass Spectrometry Service Centre, Swansea \\
\hline James Stygall & Cambridge Biotechnology \\
\hline Chris Sutton & Shimadzu Biotech \\
\hline Alan Taylor & School of Chemistry, University Of Edinburgh \\
\hline Yutaka Takahashi & Jeol UK Limited \\
\hline Christine Thompson & Pfizer Global R\&D \\
\hline Dave Tooth & Delta Biotechnology Limited \\
\hline Peter Watkins & ThermoFinnigan Limited \\
\hline
\end{tabular}


Table 2. continued

\begin{tabular}{ll}
\hline Name & \multicolumn{1}{c}{ Organization } \\
\hline \hline Dave Watson & Department of Pharmaceutical Sciences, University of Strathclyde \\
Paula Wiebkin & Applied Biosystems/MDS SCIEX \\
Jon Williams & Waters Corporation MS Technologies Centre, Micromass UK Limited \\
Jean-Claude Wolff & GlaxoSmithKline \\
Andrew Wright & AstraZeneca \\
Dominic Yu & Eastman Kodak Company \\
\hline
\end{tabular}

\section{TOF}

Laboratories 23, $31(b+c), 33(a+b), 36,45$, and 50b reported mean mass measurement accuracy $\leq 5 \mathrm{ppm}$ (Figure 3). Instrument resolving power (FWHM) ranging from 3000 to 6000 was reported. Only laboratory $50 \mathrm{~b}$ recorded all measurements in a single day. Laboratories 23, $31(b+c), 36,45$, and 50b used a lock (reference) mass to make the measurements and this was introduced using a separate sprayer, mixed with the sample or dissolved in the mobile phase. The lock (reference) mass ions employed for the measurement were in the range $\mathrm{m} / \mathrm{z} 377$ to 556 and the analyte to lock (reference) mass ion abundance ratio lay between 0.4:1 and 5:1. An external calibration was carried out on the same day as the measurement or up to six weeks prior to the measurement. Laboratories 1, 23, 31b, $33(\mathrm{a}+\mathrm{b})$, and 45 also reported mean mass measurement accuracy of $<5 \mathrm{ppm}$ or $<10 \mathrm{ppm}$ for the $\mathrm{m} / \mathrm{z} 406$ ion recorded simultaneously with the $m / z 476$ ion (Figure 4 ).

Laboratories $33 \mathrm{a}$ and $33 \mathrm{~b}$ reported the measurement of both the $\mathrm{m} / \mathrm{z} 476$ ion and the $\mathrm{m} / \mathrm{z} 406$ ion. A lock (reference) mass was not employed. For the data reported as $33 \mathrm{a}$, the calibrant mixture and analyte were infused alternately and a two point external calibration $(\mathrm{m} / \mathrm{z} 242$ and 690) was created, for the mass measurement. For the data reported by $33 \mathrm{~b}$ the sample was introduced using a LC separation. A single point external calibration was created using the background ion at $\mathrm{m} / \mathrm{z} 391$ eluting earlier in the total ion chromatogram.

Laboratories 1 and 25 reported mean mass measurement accuracy of between 5 and $10 \mathrm{ppm}$. Both used a lock (reference) mass ( $\mathrm{m} / \mathrm{z} 556$ and 502), with the analyte to lock mass ion abundance ratio maintained at $1: 1$ and a same day external calibration $(\mathrm{m} / \mathrm{z} 200-1200$ and $\mathrm{m} / \mathrm{z}$ $130-770)$ recorded. Therefore, there is no clear explanation for the discrepancy in accuracy of these two laboratories, when compared to the other laboratories employing very similar protocols. For example laboratories 1, 31b, and 45 all used $m / z 556.2771$ as a lock (reference) ion.

Laboratory 38 reported mean mass measurement accuracy of $36 \mathrm{ppm}$ (Figure 3). The instrument used was similar to that employed by laboratory 33, and like laboratory 33 no lock (reference) mass was used to make the measurements, but a same day external calibration procedure was employed. It has been noted that the instrument used by laboratory 38 was a much older version of that used by laboratory 33 and this may explain the differences in the data reported. Laboratory 38 reported mean mass measurement accuracy of 36 ppm for $\mathrm{m} / \mathrm{z} 498$ ion. Laboratory $47 \mathrm{~b}$ reported a mean mass measurement accuracy for the $\mathrm{m} / \mathrm{z} 498$ ion of 5 ppm (Figure 5). The measurements were made over a six day period using a two point internal calibration $(\mathrm{m} / \mathrm{z} 481$ and 525) with the internal calibrant mixed with the sample. The analyte to internal calibrant ion abundance ratio was $2: 1$.

\section{FTMS}

With the exception of laboratory 14 (2 ppm), all laboratories $(11,21 b, 26,32(a+b)$, and 57$)$ reported mean mass measurement accuracy of $\leq 1 \mathrm{ppm}$ for the measurement of the $m / z 476$ ion using ESI-FTMS (Figure 3). All laboratories used broadband detection and data set sizes varying from 64 to $512 \mathrm{~K}$. The five measurements from laboratories $32(\mathrm{a}+\mathrm{b})$ were recorded in a single day, with all other laboratories recording there data over a time period of up to 28 days. Instrument resolving power in the range 14,000 to 122,000 (FWHM) was reported.

Not all laboratories carried out an external calibration on the day of each measurement. Laboratories $21 \mathrm{~b}$ and 55 used calibrations recorded on the same day, within two days (laboratory 21b), or up to eight days previous (laboratory 55). Laboratory 57 employed an internal calibration procedure, with PEG 600 mixed with the sample. The sodium adduct ions of PEG were used to create the internal calibration.

A variety of external calibration ranges were employed and these were as narrow as $\mathrm{m} / \mathrm{z} 300-600$ (laboratory 32) and as broad as $m / z$ 322-1572 (laboratory $21 b)$. Three out of the seven labs (21b, 26, and 32b) also employed a lock (reference) mass for some or all of their measurements and the $m / z$ of the lock (reference) mass ions was as low as $m / z 348$ and no higher than $m / z 571$. Where reported, the ratio of analyte to lock (reference) mass ion abundance lay between 3:1 and 0.3:1. Only laboratory $32(\mathrm{a}+\mathrm{b})$ reported an FTMS measurement of the $m / z 406$ ion (Figure 4 ). For 32a, a same day external calibration was used with the mass measurement $(\mathrm{m} / \mathrm{z}$ $300-600)$. The data for $32 \mathrm{~b}$ used the external calibration and a lock (reference) mass $(\mathrm{m} / \mathrm{z} 437)$ correction, producing slightly improved mass accuracy. The ratio of 
analyte to lock (reference) mass ion abundance was not reported.

Laboratories $2(\mathrm{c}+\mathrm{d})$ reported the mass measurement of the $m / z 498$ ion by ESI-FTMS (Figure 5 ). The five measurements for $2 c$ were recorded in a single day, whereas the data for $2 \mathrm{~d}$ was recorded over 20 days. Both data sets used the same external calibration procedure, recorded on the day of each measurement in the range $m / z 167$ to 571 , to produce mean mass measurement accuracy of $<1$ ppm.

\section{Triple Quadrupole}

Laboratories 6 and 31d reported data from triple quadrupole instruments (Figure 3), with mean mass measurement accuracy of $1.3 \mathrm{ppm}$ for both laboratories, but with laboratory 6 showing much greater day to day variability. Instrument resolving power ranging from 2000 to 3100 (FWHM) was reported. An external calibration just prior to the mass measurements was not recorded by either laboratory and this was probably not required due to the inherent long term external calibration stability of triple quadrupole mass spectrometers. Both laboratories employed an internal calibration protocol using multiple ions (a minimum of 5) in the range $\mathrm{m} / \mathrm{z} 402$ to $\mathrm{m} / \mathrm{z} 525$ and where reported the analyte to internal calibrant ion abundance ratios were in the range $0.4: 1$ to $2.8: 1$.

\section{Magnetic Sector}

Laboratories $15 \mathrm{a}$ and $34(\mathrm{c}+\mathrm{d})$ reported the mass measurement of the $\mathrm{m} / \mathrm{z} 476$ ion using a magnetic sector field instrument and ESI (Figure 3). Both 15a and 34d used the peak matching technique and the ion at $\mathrm{m} / \mathrm{z}$ 448 from polyethyleneimine and reported mean mass accuracy measurements of $\leq 1.0 \mathrm{ppm}$. The ratio of the analyte to lock (reference) mass ion abundance was reported as $0.5: 1$ by laboratory $34 \mathrm{~d}$. Laboratory $34 \mathrm{~d}$ recorded all data in a single day and 15 a made measurements over a six week period.

Laboratory $34 \mathrm{c}$ employed the accelerating voltage scanning method using a narrow range internal calibration to make the measurements $(m / z 448,491)$ with the ratio of the analyte to the internal calibrant ion abundance between 1:5 and 1:10. Mass measurements were made over a two day period and the mean mass measurement accuracy was $<1$ ppm.

\section{The Measurement of the $\mathrm{m} / \mathrm{z} 498$ Ion by MALDI-MS}

Six laboratories reported data recorded using MALDI combined with TOF, FTMS and quadrupole-TOF (Figure 5). Laboratories 18, 28b, and 47a employed an internal calibration protocol with TOF to record the measurements with mean mass accuracy of $1.4 \mathrm{ppm}, 22$ ppm, and 2.0 ppm respectively. The internal calibrant, sample and matrix were mixed and cocrystallized on the MALDI target. Where the information was provided, the ratio of the analyte and internal calibrant ion abundance ranged from 2:1 through to 1:4. The internal calibration was carried out across a narrow range of $\mathrm{m} / \mathrm{z}$ values centred on $\mathrm{m} / \mathrm{z} 498$ (laboratories 18 and 47a used $\mathrm{m} / \mathrm{z} 481$ and 525 from poly(ethylene glycol), laboratory $28 \mathrm{~b}$ used ions generated from $\alpha$-lactose and lactodifucotetraose in the range $m / z 300-700$ ). The measurements were made either on a single day ( $28 \mathrm{~b}$ and 18$)$ or over three days (47a).

Laboratories 31e and 32d employed a different approach. An external calibration was recorded followed by the mass measurement of the $m / z 498$ ion using a single lock (reference) mass correction $(\mathrm{m} / \mathrm{z} 556$ for laboratory $31 \mathrm{e}, m / z 525$ for laboratory $32 \mathrm{~d}$ ). The sample, the matrix, and the lock (reference) mass compound were cocrystallized on the MALDI target. Mean mass measurement accuracy of $2 \mathrm{ppm}$ and $0.6 \mathrm{ppm}$ was reported. Laboratory 32c also reported data using a procedure where the measurement was made on the same day as an external calibration over the range $\mathrm{m} / \mathrm{z}$ $400-700$, but without the use of a lock (reference) mass to produce accuracy of $<2$ ppm. Laboratory 32 used broadband detection and data set size of $512 \mathrm{~K}$.

An overall summary of the results is shown in Table 1 , which shows the percentage of mean mass measurements that correspond to three criteria of mass accuracy. The table compares the mean mass measurement accuracy achieved by each instrument and type of experiment.

\section{Conclusions}

The report summarizes the data from a unique exercise to evaluate the relative capabilities of the current range of mass spectrometers and the protocols used to record accurate mass measurements of small molecules. The study involved the accurate mass measurement of a mono-isotopic ion from a single component sample, with no interfering ions present from the sample. At this stage, the data evaluation has been based solely on the mean mass measurement accuracy. In nearly all cases, the measurements did not involve a chromatographic introduction.

Magnetic sector field mass spectrometers have traditionally been used to record accurate mass measurements. Of the three common techniques reported, peak matching and dynamic voltage scanning clearly produced the most accurate results, with 88 and $60 \%$ of mean mass measurements $\leq 1$ ppm respectively. Only $50 \%$ of the laboratories that employed dynamic magnet scanning experiments reported data with mean mass accuracy of $\leq 10 \mathrm{ppm}$. The results clearly reflect the accepted capability of the three techniques. For FTMS, $83 \%$ of laboratories reported mean mass measurement accuracy of $\leq 1 \mathrm{ppm}$. This result is comparable to that from peak matching and superior to that recorded using dynamic voltage scanning and meets the expec- 
tation of the capability of FTMS for accurate mass measurements.

Of the low-medium resolution mass spectrometers, quadrupole-TOF produced the highest accuracy measurements, with $83 \% \leq 5 \mathrm{ppm}$, compared to $65 \%$ from TOF. This may be due as much to the optimization of current protocols as to any deficiency in instrument capability. The capabilities of both TOF and quadrupole-TOF mass spectrometers have only recently been enhanced to allow accurate mass measurements to be recorded. Therefore, further investigation of the protocols used to record accurate mass measurements is necessary to further develop the capability of these two types of mass spectrometer.

To summarize the performance of the medium and high resolution instruments, magnetic sector field mass spectrometers used in peak matching mode and FTMS were capable of the highest mass accuracy ( 83 and $88 \%$ $\leq 1 \mathrm{ppm}$ ). Quadrupole-TOF and TOF instruments generally achieved mean mass measurement accuracy between 5 and $10 \mathrm{ppm}$. In many cases an identical protocol and mass spectrometer were used to make the mass measurements, but an equivalent performance was not reported. This illustrates the need for guidance on the appropriate application of the various protocols employed.

Low resolution instruments have not traditionally been employed to record accurate mass measurements, but both triple quadrupoles used in the inter-comparison produced mean mass measurement accuracy of $<2$ $\mathrm{ppm}$. The result illustrates the capability of low resolution mass spectrometers when a sound experimental protocol is used and there are no unresolved isobaric ions. But it should be noted that only two labs reported triple quadrupole data.

The precision of the data from each instrument and experiment type was not discussed in detail in this report, but is an important consideration when evaluating their relative capabilities. Therefore, a thorough statistical evaluation of the data is underway and will be reported in a subsequent publication. Such statistical evaluation of accurate mass measurement data has received limited coverage in the literature. One key exception is the report by Sack and coworkers, who proposed a method for statistical evaluation of accurate mass measurement quality for double-focusing magnetic sector mass spectrometers [24]. Such statistical understanding is important, as it will reduce the uncertainty when selecting the elemental formulae that result from accurate mass measurement.

The data is useful to both novice and experienced users for a simple comparison of the capability of each instrument type. A detailed discussion of the resolving power of each instrument has not been included as the intercomparison was designed so that this parameter would have minimal impact on the results (no interferences in the sample). If an interfering ion had been present, mass spectral resolution would become a significant factor and would be reflected in the data produced by each type of mass spectrometer. It is envisaged that the effect of this will be investigated in a further intercomparison.

In addition, a best practice guide for accurate mass measurement is currently being prepared. It was agreed that the guide should be complemented with sound experimental data to give further credibility to the suggested approaches. Therefore, the data from the intercomparison will be used in the development of the guide. In many cases the reported mass accuracy reflected the capability of each type of mass spectrometer, but there were a significant number of exceptions. Also, mass spectrometers of the same type often produced equivalent mean mass measurement accuracy, but with significant differences in precision. From the intercomparison data we will be able to evaluate those protocols that produced excellent accuracy and precision for each type of mass spectrometer. These protocols can then be compared with those described by laboratories that produced poorer accuracy and/or precision. The key points for best practice will then be established from this comparison for each type of mass spectrometer and accurate mass measurement technique.

\section{Acknowledgments}

Additional contributions to this paper were made by Mike Sargent and Rita Harte at LGC. The authors are grateful to all the participants in the intercomparison and a number of people who have provided technical advice for the study, they are listed in Table 2. The authors also thank Alan Payne at Kodak Research and Development, Harrow, UK for providing the compound circulated for the intercomparison. The work described in this paper was supported under contract with the Department of Trade and Industry of the United Kingdom as part of the National Measurement System Valid Analytical Measurement (VAM) program.

\section{References}

1. Gross, M. L. Accurate Masses for Structure Confirmation. J. Am. Soc. Mass Spectrom. 1994, 5, 57.

2. Price, P. C.; Gale, P. J.; Loo, J. A.; Heller, D. N.; Richardson, S. D. Duncan, M. W. ASMS Guidelines for Exact Mass Measurement and Elemental Composition-New Perspectives. Proceedings of the 50th Annual Conference on Mass Spectrometry and Allied Topics; Orlando FL, 2002.

3. Aston, F. W. Bakerian Lecture-A New Mass-Spectrograph and the Whole Number Rule. Roy. Soc. Proc. A. 1927, 115, 487-514.

4. Beynon, J. H. High Resolution Mass Spectrometry of Organic Materials. Adv. Mass Spectrom. 1959, 328-354.

5. Quisenberry, K. S.; Scolman, T. T.; Nier, A. O. Atomic Masses of $\mathrm{H}^{1}, \mathrm{D}^{2}, \mathrm{C}^{12}$, and $\mathrm{S}^{32}$. Phys. Rev. 1956, 102(4), 1071-1075.

6. Nier, A. O. In Improvements in Double-Focusing Mass Spectrometry. Nuclear Masses and Their Determination; Hintenberger, H., Ed.; Pergamon Press: London, New York, Paris, Los Angeles, 1957; pp 185-193.

7. Craig, R. D.; Green, B. N.; Waldron, J. D. Application of High Resolution Mass Spectrometry in Organic Chemistry. Chimia 1963, 17, 33-42.

8. Bieman, K. Utility of Exact Mass Measurement. Methods Enzymol. 1990, 193, 295-305. 
9. Bieman, K. High-Resolution Mass Spectrometry of Natural Products. Pure Appl. Chem. 1964, 9(1), 95-118.

10. Campbell, A. J.; Halliday, J. S. Mass Measurement from Low Intensity Ion Beams. Proceedings of the 13th Annual Conference on Mass Spectrometry and Allied Topics; St. Louis, MO, May 1965; pp 200-203.

11. Green, B. N.; Merren, T. O.; Murray J. G. Fast Scanning of Mass Spectra at High Resolution. Proceedings of the 13th Annual Conference on Mass Spectrometry and Allied Topics; St. Louis, MO, May 1965; pp 204-211.

12. Marshall, A.G. Scaling MS Plateaus with High-Resolution FT-ICRMS. Anal. Chem. 2002, 74(9), 253A-259A.

13. Marshall, A. G.; Hendrickson, C. L.; Jackson, G. S. Fourier Transform Ion Cyclotron Resonance Mass Spectrometry: A Primer. Mass Spectrom Rev. 1998, 17, 1-35.

14. Amster, I. J. Fourier Transform Mass Spectrometry. J. Mass Spectrom. 1996, 31, 1325-1337.

15. Eckers, C.; Wolff, J. C.; Haskins, N. J.; Sage, A. B.; Giles, K.; Bateman, R. Accurate Mass Liquid Chromatography/Mass Spectrometry on Orthogonal Acceleration Time-of-Flight Mass Analyzers Using Switching between Separate Sample and Reference Sprays. 1. Proof of Concept. Anal. Chem. 2000, 72, 3683-3688.

16. Wolff, J. C. Eckers, C.; Sage, A. B.; Giles, K.; Bateman, R. Accurate Mass Liquid Chromatography/Mass Spectrometry on Quadrupole Orthogonal Acceleration Time-of-Flight Mass Analyzers Using Switching between Separate Sample and Reference Sprays. 2. Applications Using the Dual-Electrospray Ion Source. Anal. Chem. 2001, 73, 2605-2612.

17. Blom, K. F. Estimating the Precision of Exact Mass Measurements on an Orthogonal Time-of-Flight Mass Spectrometer. Anal. Chem. 2001, 73, 715-719.

18. Richards, D. Accurate Mass Quadrupole Time-of-Flight Mass Spectrometry for Veterinary Drug Metabolism. Proceedings of the 25th Annual Meeting of the British Mass Spectrometry Society; University of Southampton, UK, 2001; p 78; and personal communication, 2001.

19. Haddon, W. F.; Lukes, H, C. Elemental Composition Mass Spectrometry. Proceedings of the 22nd ASMS Conference on Mass Spectometry and Allied Topics; Philadelphia, PA, 1974; pp 436-439.

20. Haddon, W. F.; Harden, L. A.; Lieberman, A. E. Accurate Mass Measurement by Quadrupole Mass Spectrometry; Applications to LSIMS and Particle Beam-EI Spectra. Proceedings of the 38th ASMS Conference on Mass Spectometry and Allied Topics; Tucson, AZ, 1990; pp 122-123.

21. Tyler, A. N.; Clayton, E.; Green, B. N. Exact Mass Measurement of Polar Organic Molecules at Low Resolution Using Electrospray Ionization and a Quadrupole Mass Spectrometer. Anal. Chem. 1996, 68, 3561-3569.

22. Stafford, G. J., Jr. Ion Trap Mass Spectrometry: A Personal Perspective. J. Am. Soc. Mass Spectrom. 2002, 13(6), 589-596.

23. Sargent, M.; O'Connor, G. Feasibility Study: Mass Spectrometry Techniques for Accurate Molecular Weight Determinations of Large Molecule; 2002. Reference number LGC/VAM/2001/026. Available from the author upon request.

24. Sack, T. M.; Lapp, R. L.; Gross, M. L.; Kimble, B. J. A Method for the Statistical Evaluation of Accurate Mass Measurement Quality. Int. J. Mass Spectrom. Ion Processes. 1984, 61, 191-213.

\section{Appendix-The Intercomparison Questionnaire}

\section{Background and Aims}

A feasibility study carried out in 2001 highlighted the rapid growth in the use of accurate mass measurements in the chemical and biochemical industries. The pace at which this new technology has been implemented has outpaced the development of expertise to adequately validate methodology that is now in routine use. This is particularly serious because complex instrumentation is being introduced into groups with little background experience in mass spectrometry.

This collaborative study will focus on small molecules $(<1000 \mathrm{a} \mu)$ and the experimental approach is designed to evaluate the variation in accurate mass measurement across a broad range of instrument types. The resulting data will be used to prepare guidance on undertaking key aspects of the methodology in order to obtain robust measurements and traceable data.

\section{Implementation of Study}

1. LGC to provide suitable compound to collaborators, along with details of solubility and questionnaire to be completed with the experimental work.

2. Collaborators to mass measure the molecular ion of the test compound using local protocols.

3. Mass measurement and completed questionnaire to be returned to LGC for data evaluation.

\section{Sample Preparation}

1. $50 \mathrm{mg}$ of solid material supplied.

2. Analyze directly by ionization technique of choice.

3. For liquid introduction methods, a sample solution can be prepared by dissolving the solid in a few drops of tetrahydrofuran and diluting to the required volume with methanol.

4. Suggested MALDI sample preparation, but please use local sample preparation method if preferred.

Sample solution. $5 \mathrm{mg} / \mathrm{mL}$ in $\mathrm{MeOH}(\sim 200 \mathrm{uL} \mathrm{MeCl} 2$ added) sample dissolves slowly.

Matrix solution. $38.5 \mathrm{mg} / \mathrm{mL} \mathrm{MeOH}$ 2,5-dihydroxybenzoic acid (DHB) $(0.25 \mathrm{M})$. Mix $70 \mathrm{uL}$ of matrix solution with $10 \mathrm{uL}$ of sample solution. Dispense $0.5 \mathrm{uL}$ of mixture onto sample plate and allow to air dry.

\section{Instrumentation}

Please complete a separate questionnaire for each instrument used and return electronically where possible.

\section{Accurate Mass Measurement}

In positive ion mode, make five replicate mass measurements of the molecular ion of this compound, nominal mass $475,[\mathrm{M}+\mathrm{H}]^{+} 476$ using locally accepted protocols. Five separate sample introductions should be used, ideally on separate days.

\section{Additional Experimental Work (Optional)}

If time is available make additional mass measurements of one or more fragments of molecular ion (EI, MS/MS, in-source CID). Please provide five replicate measurements of each species. 


\section{Results}

\section{Molecular Ion}

List the five replicate measurements of the molecular ion in the table below, along with the date and time of the measurement and the associated instrument calibration.

\section{Fragment Ions}

Please insert an additional copy of the table for each fragment ion and list the five replicate measurements.

Discarded Measurements

\begin{tabular}{|c|c|c|c|}
\hline Replicate & $\begin{array}{c}\text { Mass } \\
\text { Measurement }\end{array}$ & \multicolumn{2}{|c|}{ Date and Time } \\
\hline & & Calibration & Mass Measurement \\
\hline 1 & & & \\
\hline 2 & & & \\
\hline 3 & & & \\
\hline 4 & & & \\
\hline 5 & & & \\
\hline
\end{tabular}

Please state if any measurements were discarded and if so give the reasons why.

\section{Experimental Questions}

1. Make and model of instrument used.

2. Ionization technique used (for MALDI and LSIMS, describe the sample preparation).

3. Indicate the method of sample introduction in the table below.

4. Measurement methodology.

(a) Number of scans averaged to obtain each mass measurement.

\begin{tabular}{|c|c|}
\hline LC & \\
\hline GC & \\
\hline Solids Probe (+ probe temperature) & \\
\hline CE & \\
\hline CEC & \\
\hline Syringe pump infusion & \\
\hline Other & \\
\hline
\end{tabular}

(b) For magnetic sector users, did you use static peak matching or dynamic scanning?

(c) For FTMS users indicate the storage time for acquisition, broadband or narrow band acquisition, data set size and magnet size.

(d) Please provide a typical spectrum from the experiments where possible.

5. Software used and generation.

6. Calibration details.

- Calibration range

- Calibrant used

7. Reference/lock mass details.

- Was a reference/lock mass ion(s) used to make the measurements?

- If YES, what species was employed and specify the $\mathrm{m} / \mathrm{z}$ value of the ion/ions used.

- Describe the method of introduction of the reference ion(s) (e.g. lock spray, post column addition, leak valve, etc.).

- Absolute intensity/abundance of analyte and reference ion for each measurement.

8. Give an estimate of your expected (day to day) mass accuracy (mMU).

9. Instrument resolution when carrying out the mea-

\begin{tabular}{|c|c|c|}
\hline Measurement & Analyte Ion Intensity & Reference Ion Intensity \\
\hline 1 & & \\
\hline 2 & & \\
\hline 3 & & \\
\hline 4 & & \\
\hline 5 & & \\
\hline
\end{tabular}

surement (state the resolution definition used for the calculation).

\section{Participant Details}

\begin{tabular}{|l|l|}
\hline Name & \\
\hline Organisation & \\
\hline Email address & \\
\hline Phone number & \\
\hline Lab reference number & \\
\hline
\end{tabular}

